\title{
Structure of d-Urobilin
}

\author{
By S. Derek Killilea and Pádraig O'Carra \\ Department of Biochemistry, University College, Galway, Irish Republic
}

(Received 14 August 1972)

d-Urobilin is the term originally given to a dextrorotatory urobilin isolated from the faeces of patients undergoing treatment with broad-spectrum antibiotics (Sborov et al., 1951; Watson \& Lowry, 1956). The same pigment was subsequently demonstrated in the faeces of a patient suffering from thalassaemia (Gray \& Nicholson, 1957). The properties and reactions of this urobilin led Gray \& Nicholson (1958) to propose that it contained one double bond more than the optically inactive i-urobilin obtainable synthetically and also from the faeces of some individuals. Structure (I) (Scheme 1) had been well established for i-urobilin, and Gray \& Nicholson (1958) proposed that the extra unsaturation in d-urobilin resided either in a vinyl side chain on one of the end rings, as in structure (II), or as an extra double bond within one of these end rings. The vinyl side chain has been strongly favoured in the considerable subsequent discussion on the subject (see, e.g., Moscowitz et al., 1964; Jackson et al., 1966; Moscowitz, 1967).

Our results, however, indicate that d-urobilin, prepared in the classical manner, contains neither a vinyl side chain nor 'extra unsaturation' of any sort.

\section{Material and methods}

Samples of d-urobilin were isolated as described by Watson \& Lowry (1956) from the faeces of patients who had been treated with broad-spectrum antibiotics (mainly tetracycline). Preparation of other bilins followed established procedures (see Gray et al., 1961).

Chromic acid degradation of bilins and t.l.c. identification of the imide products were performed as described by Rudiger (1969) and Rudiger \& O'Carra (1969), with the modification that the silica gel $\mathrm{G}$ plates were subjected to multiple development (three to six times) with the solvent carbon tetrachloride - ethyl acetate - cyclohexane (32:9:4, by vol.). This greatly improved the resolution of methylvinylmaleimide (III) from ethylmethylmaleimide (IV). The imides were initially detected by the benzidine method (Rudiger, 1969; Rudiger \& O'Carra, 1969) but subsequently by the safer starchiodide method (Killilea \& O'Carra, 1971). Comparative t.l.c. of bilins was performed as described by Rudiger \& O'Carra (1969) and O'Carra \& Killilea (1970).

\section{Results and discussion}

Some doubt about the presence of a vinyl side chain in d-urobilin was expressed by O'Carra et al. (1964), who noted that d-urobilin did not react with cysteine or other thiol-containing compounds which readily add across vinyl side chains of other bilins, forming thioether adducts. This observation we could readily confirm.

The sensitive chromic acid degradative method developed for bilins by Rudiger $(1968,1969)$ releases vinyl-bearing rings, similar to that of structure (II), in the form of methylvinylmaleimide (III), which can be readily distinguished by t.l.c. methods (see above) from ethylmethylmaleimide (IV), formed under the same conditions from ethyl-bearing rings. The two central propionic acid-bearing rings appear as haematinic acid (V).

In model experiments this technique was applied to test mixtures of bilins containing various proportions of vinyl-bearing and ethyl-bearing rings. The expected maleimides could invariably be identified unambiguously among the products, and a semiquantitative estimate of the proportions of the various rings could be obtained by visual inspection of the t.l.c. plates, due allowance being made for the lower yield of the more unstable vinyl derivative (about one-quarter that of the ethyl and propionate derivatives).

When the technique was applied to preparations of d-urobilin no trace of a vinyl-bearing imide could be detected, even when the amount of bilin degraded was increased to the saturation point of the technique. The estimated ratio of ethyl-bearing to propionatebearing imides (compound IV: compound V) was the same for d-urobilin as for i-urobilin and was approx. 1:1. This indicates further that d-urobilin contains two ethyl-bearing rings and no vinyl group.

The production of a red pigment by alkali treatment of d-urobilin (Gray \& Nicholson, 1958) has previously been attributed to isomerization of the putative 'extra double bond' into a bridge position, to form a mesobiliviolin-type pigment (structure VII). Since mesobiliviolin $\left(\mathrm{H}_{40}\right)$ is one oxidation level above i-urobilin $\left(\mathrm{H}_{42}\right)$, this isomerization was taken

Vol. 129 


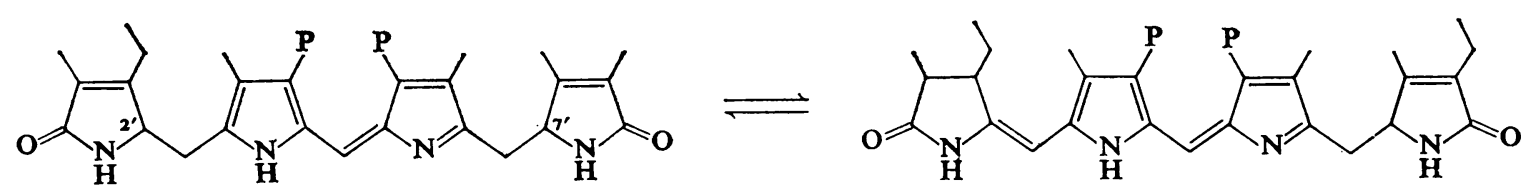

(I) (i-Urobilin)<smiles>C=CC1=C(C)C(=O)NC1Cc1[nH]c(/C=C2\N=C(CC3NC(=O)C(CC)=C3C)C(C)=C2P)c(P)c1C</smiles>

(II)<smiles>C=CC1=C(C)C(=O)NC1=O</smiles>

(III)<smiles>CCC1=C(C)C(=O)NC1=O</smiles>

(IV)<smiles>CC1=C(P)C(=O)NC1=O</smiles>

(V)

(VI) (Mesobilirhodin)<smiles>CCCCCCCCCCCCCC</smiles><smiles>CCC1=C(C)/C(=C/c2[nH]c(/C=C3/N=C(CC4NC(=O)C(CC)=C4C)C(P)=C3C)c(P)c2C)NC1=O</smiles>

(VII) (Mesobiliviolin)

$$
\downarrow-\mathrm{H}_{2}
$$<smiles></smiles>

(VIII) (Mesobiliverdin)

Scheme 1. $i$-Urobilin and related compounds

Abbreviation: $\mathrm{P},-\mathrm{CH}_{2}-\mathrm{CH}_{2}-\mathrm{CO}_{2} \mathrm{H}$.

as strong supporting evidence for a difference of oxidation level between d-urobilin and i-urobilin (Gray \& Nicholson, 1958). However, control experiments, checking the response of $\mathrm{i}$-urobilin to this alkali treatment, do not seem to have been performed. We find that i-urobilin undergoes a very similar conversion in alkali into a red pigment which we have equated with mesobilirhodin, an elusive bilin that had been reported sporadically in the literature from a variety of obscure sources (see O'Carra et al., 1964), but was very poorly characterized until recently. The structure of mesobilirhodin has now been established rigorously as formula (VI). It is isomeric with i-urobilin, into which it is freely and reversibly convertible (O'Carra \& Killilea, 1970; Rudiger et al., 1970). We have carefully compared this mesobilirhodin isomeride of $i$-urobilin with the red pigment formed by alkaline treatment of d-urobilin, and we can detect no difference of any sort between the two pigment preparations as regards spectral properties, reactions, degradation products or chromatographic properties of the di- methyl esters in a variety of t.l.c. systems (see O'Carra \& Killilea, 1970).

The conclusion seems inescapable that d-urobilin isomerizes to mesobilirhodin and so must be isomeric with i-urobilin.

i-Urobilin has been shown to be a racemic mixture (see Watson et al., 1966), and it now seems clear that the 'classical' preparations of d-urobilin represent a dextrorotatory component of this mixture, rather than a chemically distinct type of urobilin. The existence of a dextrorotatory $\mathrm{H}_{42}$ urobilin has been recognized for some time (see Watson et al., 1968). It has generally been termed d-urobilin- $\mathrm{H}_{42}$ to distinguish it from the 'classical' dextrorotatory pigment, which has correspondingly been distinguished as d-urobilin- $\mathrm{H}_{40}$. The possible identity of the two does not seem to have been seriously considered, in spite of the close spectral and optical similarities. We find the two to be chromatographically indistinguishable as the dimethyl esters in the t.l.c. systems of O'Carra \& Killilea (1970) and Stoll \& Gray (1970), which very easily separate other bilins differing by 
only one double bond. Further, the rapid dehydrogenation of d-urobilin to mesobiliverdin (VIII), formerly ascribed to a facilitating isomerization of the putative 'extra double bond', is exactly mimicked by the dextrorotatory $\mathrm{H}_{42}$ urobilin and seems to be due to a steric effect of the asymmetric centres, which are also the sites of dehydrogenation (positions $2^{\prime}$ and $7^{\prime}$ of structure I).

The urobilins have been extensively studied by mass spectrometry, but the results are greatly complicated by the tendency of the pigments to undergo redox disproportionations in the mass spectrometer, resulting in multiple peaks spaced $2 \mathrm{~m} / \mathrm{e}$ units apart near the presumed true molecular-ion position (see, e.g., Jackson et al., 1966; Lightner et al., 1969). Thus, although preparations of d-urobilin have been reported to yield a major peak at $m / e 588$ (correct for structure II), a less-intense peak in the same position is commonly observed with preparations of $i$-urobilin. This peak has been shown to be due to the artifact mesobiliviolin (structure VII) formed in the mass spectrometer by spontaneous dehydrogenation of the urobilin (Lightner et al., 1969). Since the dextrorotatory urobilin undergoes such dehydrogenation much more rapidly than the other component(s) of i-urobilin, it is not surprising that the $m / e 588$ peak is more intense for d-urobilin than for i-urobilin, and it is unlikely that any other significance need be attached to it.

Elemental analyses and quantitative hydrogenations constituted the original basis for the proposal of the $\mathrm{H}_{40}$ structure for d-urobilin (Watson \& Lowry, 1956; Gray \& Nicholson, 1958). However, elemental analyses of bilins are frequently unreliable, owing mainly to persistent solvent occlusion (see, e.g., Rudiger, 1967), and the difference between $\mathrm{H}_{40}$ and $\mathrm{H}_{42}$ must be considered outside the bounds of reliability. Interpretation of the quantitative hydrogenations (Gray \& Nicholson, 1958) was based on the assumption that uptake of hydrogen occurred only at bridge positions and vinyl groups or their equivalent. It is now known that end rings such as those of i-urobilin are also reduced, although more slowly, during the Pd-catalysed hydrogenation procedure (see, e.g., Kay et al., 1963), and it is our experience that the rate of such 'over-reduction' is to some extent dependent on the stereochemistry of the asymmetric centres $\left(2^{\prime}\right.$ and $7^{\prime}$ in structure I) in much the same way as the dehydrogenation of the adjacent bridge positions, discussed above. Such differential over-reduction, coupled with the possibility that the i-urobilin samples used for comparative purposes may have contained a proportion of already over-reduced material (i.e. half-stercobilin; see Watson et al., 1968; Stoll \& Gray, 1970), could account for the reported differential uptake of hydrogen by d-urobilin relative to i-urobilin.

With regard to current views on the physiology of intestinal bilin metabolism, the elimination of the $\mathrm{H}_{40}$ urobilin, represented by structure (II), creates no great problem. Indeed, such a compound has become increasingly difficult to integrate into metabolic schemes. The evidence has long indicated that both of the vinyl groups undergo reduction in the initial steps of the sequence of intestinal bacterial reductions leading from bilirubin to urobilin and stercobilin. In most metabolic schemes (see, e.g., Gray, 1963; With, 1968; Moscowitz et al., 1970) a branch pathway is postulated to provide for the retention of a vinyl group, or equivalent 'extra unsaturation', down to the urobilin stage. No direct evidence for such a branch pathway has ever been adduced and the above conclusions seem to render it unnecessary.

We thank the Medical Research Council of Ireland for financial assistance.

Gray, C. H. (1963) Compr. Biochem. 9, 101-103

Gray, C. H. \& Nicholson, D. C. (1957) Nature (London) 180, 336-337

Gray, C. H. \& Nicholson, D. C. (1958) J. Chem. Soc. London 3085-3099

Gray, C. H., Kulczycka, A. \& Nicholson, D. C. (1961) J. Chem. Soc. London 2268-2276

Jackson, A. H., Smith, K. M., Gray, C. H. \& Nicholson, D. C. (1966) Nature (London) 209, 581-583

Kay, I. T., Weimer, M. \& Watson, C. J. (1963) J. Biol. Chem. 238, 1122-1123

Killilea, S. D. \& O'Carra, P. (1971) J. Chromatogr. 54, 284-285

Lightner, D. A., Moscowitz, A., Petryka, Z. J., Jones, S., Weimer, M., Davis, E., Beach, N. A. \& Watson, C. J. (1969) Arch. Biochem. Biophys. 131, 566-576

Moscowitz, A. (1967) Proc. Roy. Soc. Ser. A 297, 1626

Moscowitz, A., Krueger, W. C., Kay, I. T., Skewes, G. \& Bruckenstein, S. (1964) Proc. Nat. Acad. Sci. U.S. 52, 1190-1194

Moscowitz, A., Weimer, M., Lightner, D. A., Petryka, Z. J., Davis, E. \& Watson, C. J. (1970) Biochem. Med. 4, 149-164

O'Carra, P. \& Killilea, S. D. (1970) Tetrahedron Lett. 4211-4214

O'Carra, P., Ó hEocha, C. \& Carroll, D. M. (1964) Biochemistry 3, 1343-1350

Rudiger, W. (1967) Hoppe-Seyler's Z. Physiol. Chem. 348, 1554

Rudiger, W. (1968) Biochem. Soc. Symp. 28, 121-130

Rudiger, W. (1969) Hoppe-Seyler's Z. Physiol. Chem. 350, 1291-1300

Rudiger, W. \& O'Carra, P. (1969) Eur. J. Biochem. 7, 509-516

Rudiger, W., Kost, H. P., Budzikiewicz, H. \& Kramer, V. (1970) Justus Liebigs Ann. Chem. 738, 197-201

Sborov, V. M., Jay, A. R. \& Watson, C. J. (1951) J. Lab. Clin. Med. 37, 52-59

Stoll, M. S. \& Gray, C. H. (1970) Biochem. J. 117, 271290 
Watson, C. J. \& Lowry, P. T. (1956) J. Biol. Chem. 218, 633-639

Watson, C. J., Moscowitz, A., Lightner, D., Krueger, W. C. \& Weimer, M. (1966) J. Biol. Chem. 241, 50375043
Watson, C. J., Lightner, D. A., Moscowitz, A., Davis, E., Petryka, Z. J. \& Weimer, M. (1968) Proc. Nat. Acad. Sci. U.S. 61, 223-228

With, T. K. (1968) Bile Pigments, pp. 146-147, Academic Press, New York 